\title{
La innovación social como herramienta para hacer frente a los retos urbanos: una aproximación al proyecto MARES-Alimentación (Madrid)*
}

\author{
Carolina Yacamán-Ochoa \\ Universidad Autónoma de Madrid \\ carolina.yacaman@uam.es \\ Simón Sánchez-Moral \\ Rosa de la Fuente \\ Universidad Complutense de Madrid \\ simon.sanchez@ghis.ucm.es \\ rdelafuente@cps.ucm.es
}

Recibido: junio de 2020

Aceptado: octubre de 2020

Publicado: abril de 2021

\section{Resumen}

El proyecto MARES de Madrid, liderado por el Ayuntamiento de Madrid (2016-2019), representa una de las iniciativas de innovación social más importantes de la ciudad en los últimos años. MARES desarrolló estrategias de resiliencia urbana y económica en materia de empleo desde el enfoque de la economía social y solidaria en cinco clústeres clave (alimentación, movilidad, reciclaje, energía y economía de los cuidados) para avanzar hacia un nuevo modelo productivo de la ciudad. Analizamos el proyecto a través del estudio de caso de MARES-Alimentación, el clúster que alcanzó el mayor grado de madurez, mediante entrevistas en profundidad y encuestas presenciales. Nuestras conclusiones permiten reflexionar sobre el papel de las redes de colaboración en el ecosistema innovador creado, los diferentes perfiles y formas de proximidad entre actores, la importancia de la infraestructura urbana catalizadora de la innovación social y la compleja relación de este tipo iniciativas con las instituciones públicas.

Palabras clave: innovación urbana; economía social y solidaria; redes territoriales de conocimiento; MARES; Madrid

* Este texto forma parte del proyecto del Plan Nacional I+D+i «Atracción de talento, innovación y creatividad para el desarrollo inteligente de la ciudad-región de Madrid» (CSO201674888-C4-4-R) (AEI/FEDER, UE). Los autores agradecen a los responsables y usuarios de la iniciativa MARES-Alimentación su participación en el estudio. 
Resum. La innovació social com a eina per fer front als reptes urbans: una aproximació al projecte MARES-Alimentación (Madrid)

El projecte MARES de Madrid, liderat per l'Ajuntament de Madrid (2016-2019), representa una de les iniciatives d'innovació social més importants de la ciutat en els últims anys. MARES ha desenvolupat estratègies de resiliència urbana i econòmica en matèria d'ocupació des de l'enfocament de l'economia social i solidària en cinc clústers clau (alimentació, mobilitat, reciclatge, energia i economia de les cures) per avançar cap a un nou model productiu de la ciutat. Analitzem el projecte a través de l'estudi de cas de MARES-Alimentación, el clúster que va aconseguir el major grau de maduresa, mitjançant entrevistes en profunditat i enquestes presencials. Les nostres conclusions permeten reflexionar sobre el paper de les xarxes de col.laboració en l'ecosistema innovador creat, els diferents perfils i formes de proximitat entre actors, la importància de la infraestructura urbana catalitzadora de la innovació social i la complexa relació d'aquest tipus d'iniciatives amb les institucions públiques.

Paraules clau: innovació urbana; economia social i solidària; xarxes territorials de coneixement; MARES; Madrid

Résumé. L'innovation sociale en tant qu'outil pour faire face aux défis urbains : une approche du projet MARES-Alimentation (Madrid)

À Madrid, le projet MARES, dirigé par la mairie de Madrid (2016-2019), représente l'une des initiatives d'innovation sociale les plus importantes de la ville ces dernières années. MARES a développé des stratégies de résilience urbaine et économique en termes d'emploi à partir de l'approche de l'économie sociale et solidaire dans cinq pôles clés (alimentation, mobilité, recyclage, énergie et économie des soins) pour s'orienter vers un nouveau modèle productif de la ville. Nous analysons le projet à travers l'étude de cas de MARES-Alimentation, le cluster qui a atteint le plus haut degré de maturité, grâce à des entretiens approfondis et des enquêtes en face à face. Nos conclusions nous permettent de réfléchir sur le rôle des réseaux de collaboration dans l'écosystème innovant qui a vu le jour, les différents profils et formes de proximité entre acteurs, l'importance des infrastructures urbaines en tant que catalyseur de l'innovation sociale et la relation complexe de ce type d'initiatives avec les institutions publiques.

Mots-clés : innovation urbaine ; économie sociale et solidaire ; réseaux territoriaux de connaissance ; MARES ; Madrid

Abstract. Social innovation as a tool to overcome urban challenges: An approximation to the "MARES Alimentación" project in Madrid, Spain

The MARES project in Madrid, led by the Madrid City Council (2016-2019), is one of the most important social innovation initiatives undertaken in the city in recent years. To advance towards a new productive model for the city, MARES developed a set of strategies for urban and economic resilience strategies for employment based on the Social and Solidarity Economy approach, which are structured around five key clusters (food, mobility, recycling, energy, care economy). We analyze the project through a case study of MARES Alimentación - the cluster that achieved the highest degree of maturity-by means of indepth interviews and face-to-face surveys. Our conclusions allow us to reflect on the role of collaborative networks in the innovative ecosystem created, the different profiles and forms of proximity between actors, the importance of urban infrastructure as a catalyst for social innovation, and the complex relationship of this type of initiative with public institutions.

Keywords: urban innovation; social and solidarity economy; territorial knowledge networks; MARES; Madrid 


\section{Sumario}

$\begin{aligned} & \text { 1. Introducción } \text { 4. Discusión de resultados. El clúster } \\ & \text { 2. La innovación social como } \begin{array}{l}\text { MAR-Alimentación como laboratorio } \\ \text { de innovación social }\end{array} \\ & \begin{aligned} \text { instrumento para la transformación del } \\ \text { modelo de desarrollo urbano }\end{aligned} \begin{array}{l}\text { 5. Conclusiones } \\ \text { 3. Metodología }\end{array} \\ & \text { Referencias bibliográficas }\end{aligned}$

\section{Introducción}

En el contexto de crisis sistémica y continuada padecida en los últimos años, los estudios urbanos han centrado su atención en la capacidad de la innovación social para generar soluciones no convencionales tanto en el plano de la eficiencia económica como en otros aspectos también vinculados al desarrollo territorial, como el bienestar social, la sostenibilidad ambiental o el buen gobierno (Méndez, 2016: 16). Esto ha provocado que la innovación social se haya popularizado en el ámbito de las políticas públicas en la Unión Europea (Moulaert et al., 2007). De acuerdo con los diferentes enfoques existentes, la innovación social puede ayudar a movilizar recursos sociales - presentes sobre todo en las áreas urbanas-, a crear nuevas formas de organización en las funciones y los procesos de producción y distribución más eficientes en términos económicos y ambientales, así como a la elaboración de nuevos productos que revalorizan el capital territorial (p. ej., Martínez Moreno, 2011). Klein et al. (2014) apuntan, además, a la creación de nuevas formas más participativas de coordinación y regulación de la actividad, entre las que se menciona la coconstrucción publicoprivada de políticas públicas, mientras que Moulaert y Naussbaumer (2005) inciden en la importancia del contexto institucional como catalizador de la cooperación y la interacción de las redes de actores que impulsa el fenómeno de la innovación social. En el extremo, la innovación social puede cumplir un papel importante no solo en la crítica-oposición al capitalismo contemporáneo en sus vertientes económica, social y ambiental, sino también como instrumento para su superación a través de la construcción de un modelo económico alternativo (Fuller y Jonas, 2003; Gibson-Graham, 2008).

En el contexto de las ciudades españolas, se observa un creciente número de investigaciones que buscan explicar el fenómeno de la innovación social a partir de diferentes enfoques (Subirats y García Bernardos, 2015; Suriñach, 2017; González-Romero, 2020), entre los que destaca el estudio de las redes urbanas (Blanco et al., 2016; Salom et al., 2017) o las investigaciones más recientes centradas en las llamadas prácticas económicas alternativas (Sánchez Hernández, 2019). Entre estas últimas, sobresalen los estudios que se centran en identificar las estrategias más adecuadas para reconectar el campo con la ciudad mediante la activación de redes territoriales y nuevas formas de organización alternativas de producción y distribución de consumo (Méndez y Monteserín, 2017; Méndez, 2018; Espinosa, 2019; Yacamán et al., 2019; Yacamán et al., 2020). 
El repaso a esta literatura permite identificar algunas cuestiones abiertas donde, según los expertos, convendría ampliar la evidencia empírica, empezando por los distintos recursos necesarios para el impulso de la innovación social; el papel de la escala territorial, aspecto que se relaciona con el alcance económico y la ambición de las iniciativas; las trayectorias de vida y motivaciones de los actores participantes en las redes de colaboración; los factores internos o externos que explican el surgimiento y la continuidad de este tipo de iniciativas, o, respecto a ello, la compleja relación entre innovación social e instituciones públicas (Méndez, 2016; Sánchez Hernández, 2019).

A partir de este marco conceptual, el objetivo fundamental de nuestro trabajo es analizar una iniciativa de innovación social en el contexto de la región urbana madrileña: el proyecto MARES: Ecosistemas Urbanos Resilientes para una Economía Sostenible, liderado por el Ayuntamiento de Madrid (2016-2019). El proyecto propuso un conjunto de soluciones innovadoras para afrontar el desafío de la crisis económica, en relación con la destrucción de empleo y la situación de vulnerabilidad en los distritos del sur/sureste de la ciudad, a la vez que promovió nuevas formas de desarrollo territorial sostenible. Para ello, propuso el fortalecimiento del tejido productivo social en torno a clústeres empresariales en cinco sectores estratégicos (movilidad, alimentación, reciclaje, energía y cuidados) relacionados con los Objetivos de Desarrollo Sostenible y anclados en cuatro distritos de la ciudad de Madrid. Todos los clústeres sectoriales compartían objetivos generales y estrategias, a los cuales nos aproximamos a través del análisis en concreto del MAR de Alimentación.

MARES representa una de las experiencias de innovación social más importantes de los últimos años en la ciudad. En este sentido, interesa por las prácticas novedosas desarrolladas para el fortalecimiento del tejido económico, por el establecimiento de nuevas fórmulas de cooperación publicoprivada dentro del ecosistema innovador $y$, especialmente, por su potencial de transformación social a partir de la incorporación de los valores y principios de la economía social y solidaria (ESS, en adelante) al desarrollo territorial. En este contexto, nuestra investigación, que aspira a aportar una mirada de la iniciativa "desde dentro» a partir de la entrevista a sus principales responsables y la encuesta realizada a los usuarios, parte de tres hipótesis fundamentales:

(H1) El desarrollo de clústeres sectoriales favorece la aparición de relaciones de confianza mutua, sinergias de colaboración e intercambio de conocimiento entre actores - que pueden exhibir desigual grado de proximidad - como aspectos necesarios para la creación de redes de apoyo y cooperación entre empresas y emprendedores sociales.

(H2) El impulso de las capacidades y el conocimiento existentes en el ecosistema de innovación, junto con el marco de la ESS con una finalidad transformadora del modelo de desarrollo urbano, supone la mayor diferencia frente a otros clústeres convencionales. $\mathrm{Al}$ respecto, cabe plantear la existencia de un perfil de usuario «utilitarista» frente a otro «militante». 
(H3) El enfoque de clúster sectorial impulsado desde MARES, que concentra recursos y servicios orientados a fortalecer un sector productivo concreto (alimentación) en una infraestructura urbana específica, permite que esta se convierta en un polo $(b u b)$ de innovación económica y social para la ciudad y la región.

El artículo se organiza de la siguiente forma. En la sección que viene a continuación, repasamos los aportes teóricos de la innovación social, tanto desde el punto de vista de los recursos necesarios para su impulso como de los impactos sobre el desarrollo territorial. En el apartado tercero, se presenta el estudio de caso y se aportan los detalles metodológicos de la investigación, mientras que en el cuarto se plantea el análisis y la discusión de los resultados. El artículo termina con unas conclusiones para validar o contrastar las hipótesis de la investigación y la identificación de oportunidades y amenazas útiles para proyectos de innovación urbana de esta naturaleza.

\section{La innovación social como instrumento para la transformación del modelo de desarrollo urbano}

La innovación social ha adquirido un protagonismo sobresaliente dentro del ámbito del desarrollo territorial sostenible en las últimas décadas. Pese al carácter poliédrico del concepto, existe cierto consenso a la hora de definir la innovación social como aquella que parte de recursos sociales y que, al mismo tiempo, persigue fines sociales (Moulaert et al., 2007). Su relevancia como herramienta para la transformación del modelo de desarrollo urbano encuentra justificación en ambas dimensiones.

El repaso a los estudios de geografía económica, como el realizado por R. Méndez (2016), permite una primera identificación de una serie de recursos específicos para la innovación (en general), los cuales aparecen fuertemente concentrados en áreas urbanas (Méndez Gutiérrez del Valle et al., 2009; Méndez y Sánchez-Moral, 2011). Destaca así la contribución del capital físico (en forma de infraestructuras y equipamientos, centros de educación...), el capital humano y el capital financiero (en forma de inversiones de I+D+i, subvenciones y avales...); además del capital productivo, en relación con las dinámicas de colaboración que pueden emerger dentro del tejido productivo y la aparición de economías de aglomeración y dinámicas de aprendizaje colectivo. Así lo describen diferentes modelos territoriales de innovación (Moulaert y Sekia, 2003), como los medios de innovación (Maillat, 1992; Vázquez Barquero, 1999), los clústeres o los sistemas regionales de innovación (todos ellos estrechamente relacionados con los planteamientos del proyecto analizado). Desde el enfoque de la innovación social, se incide en el importante papel de la sociedad civil, lo que para algunos constituye una "cuarta hélice», así como del Estado y del tercer sector (fundaciones, ONG). La empresa privada no quedaría totalmente excluida, pero desde este planteamiento se presta especial atención a las empresas de la economía social u otros actores como los emprendedores sociales. En 
todos los casos, el interés se centra en aquellas dinámicas económicas fuertemente enraizadas en el territorio, preferentemente en la escala local y regional, que se contraponen a procesos de globalización y deslocalización dominantes.

Como se repite en esta literatura, las relaciones de colaboración que se tejen entre estos actores son en muchos casos informales y presentan un carácter horizontal y no jerárquico. El capital social favorece el establecimiento de relaciones de confianza trabadas por la densidad y por distintas formas de proximidad organizativa, cognitiva o cultural, además de la geográfica (Boschma, 2005), e incluso por una "proximidad construida", a partir de la participación en redes creadas que no existían previamente (Balland et al., 2015). Estas redes resultan fundamentales para el intercambio de conocimiento existente en el territorio, especialmente de formas tácitas del mismo, como recurso fundamental para la innovación. En algunos casos cabe hablar del surgimiento de "comunidades de práctica», entre quienes comparten claves y saberes (Wenger, 2001; Amin y Robers, 2008) y cuya experiencia compartida retroalimenta el proceso de aprendizaje compartido (Fløysand y Jacobsen, 2011), lo cual permite generar soluciones desde los propios agentes en función sus propias necesidades y las de la comunidad.

De la mano del debate sobre las formas de proximidad entre actores, surge la cuestión de la escala geográfica (Méndez, 2016). De un lado, se defiende la necesidad de apoyar la creación de redes de colaboración enraizadas en el territorio sirviéndose de los recursos endógenos y las capacidades locales para la creación de un entorno innovador (Guridi y Pérez de Mendiguren, 2014). En esto tendría un papel fundamental el contexto institucional —entendido no solo como organizaciones formales, sino también como el conjunto de normas de comportamiento, reglas, convenciones e incluso formas de gobernanza de tipo horizontal y participativo-, que hace posible o amplifica el «zumbido» de los actores locales y sus redes (Bathelt et al., 2004). Al mismo tiempo, la conexión de estos agentes y sus redes con flujos de conocimiento global y otros recursos procedentes del exterior apunta al carácter multiescalar de los procesos de innovación.

Por lo que respecta a los resultados, desde un enfoque económico se destaca que la innovación social responde a nuevas ideas, soluciones, productos o servicios que, de forma más eficiente que las alternativas existentes, resuelven necesidades no suficientemente satisfechas por el mercado y el Estado; también se manifiesta mediante la creación de nuevas formas de organización empresarial. Desde la ciencia política, se incide además en los cambios que la innovación social favorece en el desarrollo de programas, agencias e instituciones que pueden llevar a una mejor inclusión de grupos sociales vulnerables, así como al cambio en la dinámica de las relaciones sociales y de la distribución del poder (Martínez Moreno, 2011). Por su parte, Klein et al. (2014: 235) subrayan la creación o adopción de nuevas formas de hacer, nuevas coordinaciones y regulaciones, tales como la gobernanza distribuida, la coproducción de servicios y la coconstrucción de políticas públicas. En este aspecto, incide Fuente (2019: 28) al hablar de "cocreación de políticas públicas en el marco de la innovación 
democrática». Siguiendo nuevamente a Klein (2019), estas dimensiones se subsumirían en la capacidad de "transformación social», donde las innovaciones sociales son concebidas como el resultado de acciones colectivas que permiten rearticular la economía a partir de lo social y lo territorial. Se distinguiría así entre innovaciones sociales radicales o incrementales, en la medida en que son capaces de crear nuevas trayectorias de desarrollo o inflexiones en las mismas, a partir de la formulación de la propuesta de nuevas soluciones o arreglos en el plano institucional u organizacional, que presentan una vocación explícita de mejorar el bienestar de las personas. Todo ello se produciría mediante un proceso de adaptación y difusión de las innovaciones sociales en sucesivas etapas, desde la experimentación hasta la institucionalización. La existencia de diversos actores con intereses divergentes operando además en un marco institucional heterogéneo hace que el proceso, lejos de ser lineal, esté marcado por avances y retrocesos, y que su resultado sea impredecible.

Esa dimensión de la transformación social establece vínculos relevantes con algunas perspectivas críticas en la órbita del denominado "poscapitalismo» (Gibson-Graham, 2008; Mason, 2015). Es el caso de la llamada «economía social» y la "economía solidaria», que experimentaron un resurgimiento en Europa desde la década de los 70 del siglo xx, en relación con la emergencia de nuevas formas organizativas que buscaban mejorar la falta de empleo, la precariedad de las rentas y su distribución entre los y las trabajadoras (Pérez de Mendiguren et al., 2009; Yacamán et al., 2018). Junto con estos aspectos, la economía social se interesa en concreto por la democratización de la economía, tanto desde el punto de vista del funcionamiento interno de las empresas como hacia el exterior, así como por la redistribución social y el bienestar de la comunidad. Proporciona además un valor añadido al proceso de desarrollo local y al progreso socioeconómico, al favorecer el capital humano mediante el establecimiento de relaciones de confianza y de espíritu comunitario, el compromiso cívico y la participación en la sociedad, así como el fomento de una mayor cohesión social (CR-UE, 2002). En último término, la economía social no se limita exclusivamente a los procesos económicos desde lógicas alternativas, sino que más bien cuestiona el modelo vigente y genera soluciones sobre la base de lógicas de solidaridad, de modo que constituye un instrumento de transformación (Laville, 2009). Por su parte, la economía solidaria pone mayor énfasis en satisfacer las necesidades de las personas, en fomentar los principios éticos y solidarios y en mejorar la capacidad de organización de la sociedad civil, y no tanto en el sujeto jurídico del modelo empresarial (Yacamán et al., 2018).

A su vez, la ESS se relaciona con la llamada "economía alternativa» (Leyshon et al., 2003) y las prácticas económicas alternativas (PEA), en relación con las cuáles ha sido discutido nuestro estudio de caso en trabajos previos (Sánchez Hernández, 2019). Estas prácticas alternativas, que tienen un primer referente en los trabajos de Polanyi (1944), han despertado un interés creciente desde la Gran Recesión de 2008 (p. ej., Gibson-Graham, 2008; Conill et al., 2012; Sánchez Hernández, 2019). Siguiendo a Méndez (2016), existirían diferencias de la ESS respecto de las PEA en cuanto a la consideración del estatus jurídico 
como garantía de democracia interna, de la existencia de una lógica competitiva distinta a la convencional o de la aportación al bienestar de la sociedad. Por otro lado, no todas las prácticas solidarias conllevan necesariamente un proyecto transformador de la economía y la sociedad. Frente a esto, se reconoce en estas PEA, entendidas como una parte de la innovación social, una voluntad reparadora que intenta paliar las externalidades negativas, con una función transformadora que promueve otro modelo diferente de desarrollo (Levesque, 2011); la búsqueda de normas de funcionamiento basadas en la participación democrática y el trabajo voluntario, así como de nuevas formas de gobernanza y colaboración con las instituciones públicas (Salom et al., 2019). Esta riqueza de matices se traslada a los actores individuales, entre los que la evidencia disponible sugiere la existencia de motivaciones contrastadas, desde una aproximación a la economía alternativa como estrategia de autoempleo por parte de un perfil de trabajadores «culturalmente adaptados», frente a otros «culturalmente transformadores» que en efecto perseguirán una transformación ética y política de la sociedad (Llobera, 2013; Méndez, 2016).

\section{Metodología}

\subsection{Justificación y presentación del estudio de caso}

Nuestra investigación busca estudiar iniciativas publicoprivadas con capacidad de dinamizar procesos de cambio social en torno al desarrollo territorial y con una clara vocación de mejorar la sostenibilidad urbana. Dentro de este ámbito, se seleccionó el proyecto MARES de Madrid: Ecosistemas Urbanos Resilientes para una Economía Sostenible porque cumple con estos criterios, al desarrollar estrategias alternativas para dar respuesta a las necesidades básicas y a las problemáticas sociales derivadas de las sucesivas crisis, agravadas por las políticas de austeridad. En concreto, analizamos el clúster sectorial MAR de Alimentación, ya que era uno de los más consolidados, según la memoria final del proyecto, al haber concentrado el mayor volumen de empresas e iniciativas asesoradas, así como el mayor número de iniciativas generadoras de empleo y nivel de facturación e innovación (MARES Madrid, 2019).

MARES fue impulsado desde el Área de Economía y Hacienda del Ayuntamiento de Madrid, junto con ocho entidades vinculadas a la ESS (Dinamia, Grupo Cooperativo Tangente, Estudio SIC, Vivero de Iniciativas Ciudadanas, Ecooo, Todo por la Praxis, Agencia para el Empleo y Acción contra el Hambre), en el marco de la convocatoria europea Innovative Urban Action (IUA) 2014-2020. La IUA tiene por objeto proporcionar recursos a ciudades europeas para experimentar nuevas soluciones que aborden sus desafíos actuales. MARES apostó por el ambicioso objetivo de transformar la economía de Madrid a través de la generación y consolidación de empresas vinculadas a la ESS. Para ello, fue el propio partenariado publicoprivado el que catalizó la creación de un ecosistema de innovación social en el ayuntamiento, mientras que las entidades de economía social y solidaria y los emprendedores sociales 
involucrados en el proyecto tuvieron un papel relevante para avanzar hacia la transformación social y económica de la región.

Para entender mejor la singularidad de MARES, es necesario enmarcarlo en el contexto político-institucional del nuevo municipalismo de algunas ciudades españolas, como resultado del germen del «15-M», y de la capacidad de los activismos urbanos (Walliser y Fuente, 2018) para influir en el diseño de la agenda de los llamados «ayuntamientos del cambio» (Nel.lo, 2015; Navarro et al. 2019). Durante este periodo, Madrid consolidaba su apuesta por transformar la economía de la ciudad hacia modelos más democráticos, inclusivos, equitativos y resilientes, y situaba por primera vez la ESS en el centro de las políticas económicas. De esta forma, la mencionada área de gobierno del ayuntamiento desarrolló de forma participativa la Estrategia Municipal de ESS (2018-2025), con la colaboración de la Red de Economía Social y Solidaria (REAS) y las entidades que la componen. En el contexto de esta coyuntura social y política, MARES buscó crear un cambio de paradigma en torno a la creación de empleo. Por un lado, se desarrollaron un conjunto de actividades vinculadas con la generación y consolidación de actividad económica en los distintos sectores, con el respeto medioambiental y la igualdad de género como valores transversales; por otro, se buscó la generación de conocimiento y redes en el marco del ecosistema de innovación social.

Todos los clústeres de MARES compartían los mismos objetivos generales y las mismas estrategias para liderar procesos de innovación desde el paradigma de la ESS y con un enfoque integral que pivotaba sobre cuatro pilares fundamentalmente en el plano económico, sin olvidar los aspectos sociales y ambientales:

- La activación de procesos de recuperación, "prototipado» y codiseño de espacios públicos en desuso como laboratorios de innovación social.

- La puesta en marcha de un taller de competencias, capaz de poner en valor las estrategias de resiliencia económica de los colectivos ciudadanos.

- El impulso de procesos de innovación económica territorial que generen nuevo tejido económico y que mejoren la competitividad y sostenibilidad de las tramas productivas ya existentes en la ciudad, especialmente desde lugares periféricos o barrios desfavorecidos desde los que potenciar o liderar transformaciones territoriales (principalmente en Vallecas, Villaverde, Vicálvaro y distrito Centro).

- El estímulo de dispositivos de sensibilización, dinamización económica territorial y de cooperación entre agentes, con el fin de articular ecosistemas que permitan la diversificación productiva y la ampliación de posibilidades colectivas en materia de empleo y empleabilidad.

\subsection{Propuesta metodológica para el análisis de la iniciativa}

El desarrollo de la investigación ha seguido dos etapas. La primera consistió en el análisis de fuentes secundarias, que ha supuesto la revisión de documentos publicados en la web del proyecto MARES y diversa documentación interna 
facilitada por el equipo coordinador. La segunda etapa, desarrollada entre 2019 y 2020, combinó la realización de entrevistas semiestructuradas a agentes clave, como son el director general de Economía (2015-2019) del Ayuntamiento de Madrid, el director de MARES-Alimentación y el coordinador de los servicios específicos del proyecto, así como encuestas presenciales a las iniciativas y las personas emprendedoras participantes de los procesos de acompañamiento, asesoramiento e incubación y de otras actividades de carácter social. Además, se visitaron las instalaciones del proyecto en diferentes momentos.

Las principales cuestiones abordadas en las entrevistas estaban articuladas alrededor de los siguientes aspectos: conocer el origen y la gestación del proyecto, entender el modelo establecido por el partenariado del proyecto MARES, identificar las dimensiones relativas a la innovación social de los principales servicios y determinar los principales factores de éxito y dificultades en el desarrollo del mismo. Por su parte, las encuestas presenciales realizadas a las iniciativas que estaban utilizando los múltiples servicios de MARES-Alimentación durante la última etapa del proyecto constaron de 14 ítems, con preguntas cerradas y abiertas, orientadas a caracterizar el perfil de los usuarios, el uso de recursos y servicios, así como la percepción y las motivaciones de los usuarios. Todo ello relacionado con la hipótesis esbozada al comienzo en cuanto al uso utilitarista del espacio (aprovechamiento de un conjunto de recursos, servicios e infraestructuras), frente a una necesidad de conocimiento y creación de redes dentro del ecosistema del sector de la alimentación. Se trata, en suma, de un estudio de carácter exploratorio que toma como población universo a los usuarios y entidades que hicieron uso de los servicios y recursos del clúster MAR-Alimentación (37 representantes de un total de 55 iniciativas, según la información facilitada).

\section{Discusión de resultados. El clúster MAR-Alimentación como laboratorio de innovación social}

\subsection{Planteamiento general del proyecto}

El MAR de Alimentación tenía como objetivo fomentar un sistema alimentario sostenible, resiliente y diversificado para la ciudad y la región metropolitana madrileña. Para ello, orientó sus servicios y espacios a dar apoyo a iniciativas económicas vinculadas con la generación de una oferta y una demanda de productos alimentarios saludables, ecológicos y de cercanía, y conectando al conjunto de la cadena de valor alimentaria. De este modo, según su responsable, se aprovechaba, en primer lugar, la demanda creciente de los ciudadanos por cuidar su alimentación, seguida del mayor interés de las ciudades por desarrollar políticas agroalimentarias de calidad, muchas de ellas, como el caso de Madrid, agrupadas en torno al Pacto de Política Alimentaria Urbana de Milán ${ }^{1}$. En

1. El marco estratégico de acción del Pacto de Políticas Alimentarias Urbanas de Milán, firmado por más de 116 ciudades en el mundo, reconoce el importante papel que tienen las ciudades para lograr sistemas alimentarios más sostenibles (<http://www.foodpolicymilano. org/wp-content/uploads/2015/10/Milan-Urban-Food-Policy-Pact-_SPA.pdf $>$ ). 
Figura 1. Ecosistema de innovación social de MARES: principales recursos y redes movilizados por el proyecto

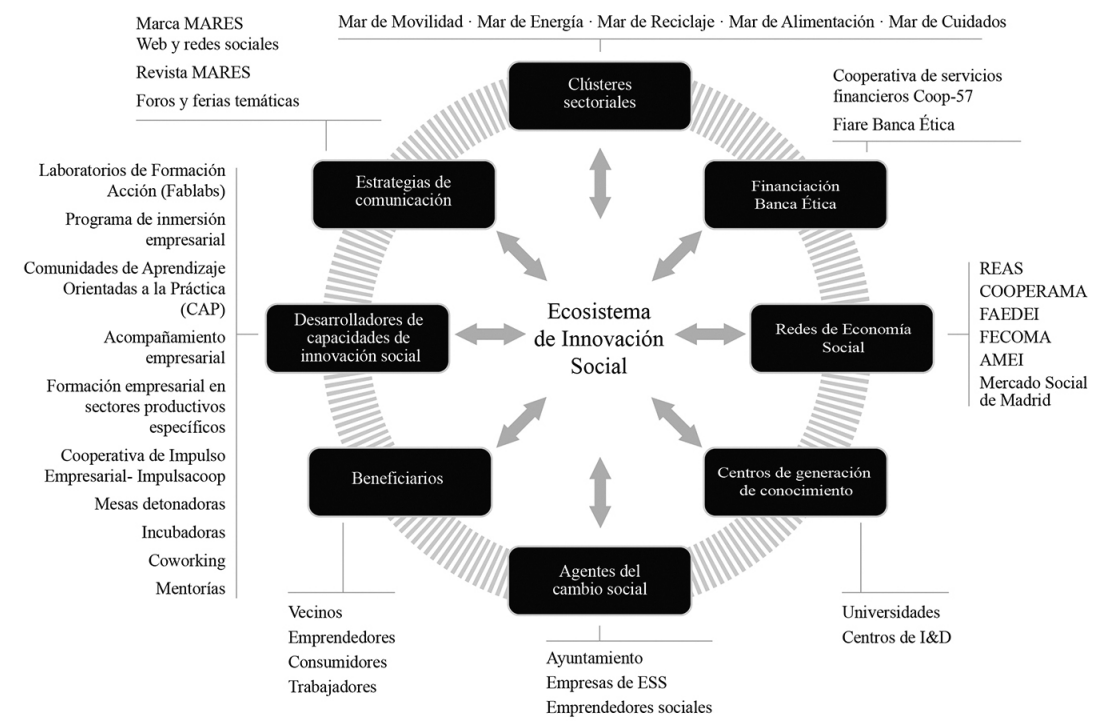

Fuente: elaboración propia, basada en el Foro Consultivo y Científico y Tecnológico (Michelini, 2019: 60).

este sentido, el diseño del proyecto respondía a la toma de conciencia sobre los impactos generados por el sistema agroalimentario globalizado, que ha provocado el deterioro de los recursos naturales, el aumento de desperdicio de alimentos, así como una evidente pérdida de rentabilidad y la desaparición de pequeñas explotaciones agrarias, y la paulatina reducción del peso del sector de la alimentación en Madrid. A partir de este contexto, se aprovechó la coyuntura social y política para convertirse en un lugar de referencia de la alimentación sostenible para el sector de la producción, la restauración y la trasformación alimentaria, la comercialización y el consumo.

La consecución de los objetivos de MARES comportaba generar un ecosistema innovador en cada uno de los clústeres sectoriales. Entre los aspectos más novedosos del ecosistema de innovación generado en MARES-Alimentación, cuyos principales elementos fueron identificados en esta investigación (figura 1), se encuentra la combinación de estrategias interrelacionadas para mejorar la sostenibilidad y la resiliencia del sistema agroalimentario. En primer lugar, se promovió la consolidación de iniciativas de producción y transformación alimentaria mediante la movilización de recursos endógenos y la puesta en valor del capital humano con que cuenta la región metropolitana madrileña. Desde esta perspectiva, el territorio adquiere un valor estratégico para mejorar el desarrollo local y deja de ser por lo tanto un espacio inerte. Esto se consiguió mediante la activación de estrategias de anclaje territorial encaminadas a la creación de valor en las cadenas productivas, frente a las tendencias pre- 
dominantes de la globalización económica que conducen al desarraigo con el territorio y a la producción de alimentos deslocalizados con las zonas de consumo próximo.

Junto con las anteriores estrategias, se impulsó la reconexión y la cooperación entre los diferentes eslabones de la cadena, principalmente fortaleciendo las alianzas entre productores y consumidores mediante el impulso de modelos cooperativos. Un ejemplo de ello fue el apoyo y acompañamiento prestados para la creación de la cooperativa de servicios Madrid Km0, integrada por transformadores de pequeña escala (cerveza, queso, repostería, vinos y aceite, etcétera). Este modelo busca solventar los problemas asociados a la distribución a través del circuito corto, mediante la gestión de un centro logístico común para mejorar los servicios de almacenamiento, preparado de pedidos y reparto bajo una marca común, lo que contribuye a mejorar la viabilidad económica de sus socios. Otro de los proyectos fue el impulso de los supermercados cooperativos La Osa (barrio de la Ventilla) y SuperCoop (barrio de Lavapiés), en la ciudad de Madrid, así como de Biolibre en Getafe. Son modelos en los que las personas son a la vez consumidoras y copropietarias, lo que les permite mejorar el acceso a la alimentación ecológica y saludable, a la vez que se avanza en la democratización del sistema agroalimentario local.

\subsection{Herramientas que componen el ecosistema de innovación social}

A partir de la identificación de los nodos, servicios y funciones dentro de los ecosistemas de MARES, podemos describir las herramientas diseñadas por el proyecto y concretar algunos ejemplos de aplicación y resultados conseguidos dentro del clúster de Alimentación.

\section{a) Las comunidades de aprendizaje orientadas a la práctica: el conocimiento como motor de cambio social}

Una de las herramientas más interesantes desarrolladas por todos los clústeres sectoriales fueron las llamadas Comunidades de Aprendizaje orientadas a la Práctica (CAP). Se basaron en una forma pedagógica innovadora, en torno a espacios de intercambio de conocimiento colectivo para aumentar las capacidades individuales y colectivas entre actores diversos, con el fin de crear soluciones adaptadas a las necesidades e intereses concretos de los integrantes en áreas temáticas y gerenciales específicas (planificación, calidad, innovación, salto de escala...). Se crearon 34 CAP que permanecieron activas durante la ejecución del proyecto. Estos espacios relacionales, los cuales interactuaban con regularidad, se orientaron fundamentalmente a fortalecer los proyectos económicos implicados y mejorar las competencias para la empleabilidad, junto con la creación de redes de conocimiento comunitario encauzadas hacia la acción. A modo de ejemplo, en el MAR- Alimentación se desarrollaron diversas CAP: la de huertas inclusivas comunitarias, la de consumo sostenible y saludable, la de supermercados cooperativos, la de gestión de cafeterías municipales y por último la de comedores ecológicos en escuelas infantiles (que destacó por con- 
tar con la participación de más de 90 profesionales entre personal docente, educadoras/es, personal de cocina, AMPAs y nutricionistas interesados en promover comedores ecológicos en escuelas infantiles). Cada CAP desarrollaba su propio plan de acción en función de la problemática identificada en cada sector y se coordinaba a su vez con los servicios específicos de acompañamiento empresarial. Los facilitadores del MARES favorecieron el nexo con el resto de CAP asegurando que el conocimiento no fuera estático y se nutriera de otros aprendizajes sectoriales, generando un ecosistema de conocimiento integral. En suma, estas redes en torno al conocimiento son un ejemplo de nuevas formas de movilización de capacidades para la acción colectiva en el seno de comunidades específicas de aprendizaje y la generación de debates en la agenda sectorial (Wenger, 2001; Méndez, 2016).

\section{b) Servicios específicos de acompañamiento empresarial: la economía al servicio de las personas}

Los servicios transversales desarrollados en MARES estuvieron orientados a dar respuesta a necesidades específicas estructurales del tejido emprendedor, a la consolidación de microempresas y a brindar formaciones ad-hoc para los diferentes modelos de negocio. Cabe destacar que no se prestó un servicio de asesoramiento convencional, sino uno integral orientado hacia el emprendimiento colectivo bajo los principios de la ESS. Para ello, se diseñó un programa gratuito y permanente de acompañamiento personalizado, transversal y sectorial, con profesionales especializados en apoyar la creación y la consolidación preferentemente de cooperativas de trabajo asociado y de servicios. Esto permitió complementar el conocimiento práctico que surgió de las diferentes CAP con el conocimiento experto, lo que contribuyó a regenerar el frágil tejido económico y social impactado por la crisis, a mejorar la viabilidad económica de las empresas participantes y a definir nuevas estrategias de futuro en torno a un desarrollo económico más sostenible y equitativo.

Otro de los servicios específicos más destacados fue Impulsacoop, creado para dar soporte a nuevos emprendedores que necesitaban iniciar una actividad económica colectiva. Este hosting jurídico se constituyó como una cooperativa integrada por varias entidades especializadas en servicios de apoyo empresarial de la ESS. El aspecto más novedoso es que ofrecía soporte empresarial completo, pues, además de brindar apoyo jurídico, daba cobertura de seguros éticos y de prevención de riesgos laborales a las nuevas entidades. Según el coordinador de los servicios específicos, «Impulsacoop ayudó a formalizar proyectos precarios y, en segundo lugar, estimuló nuevas iniciativas vinculadas con la ESS». De acuerdo con su diagnóstico, «la mayor parte de los proyectos eran de autoempleo colectivo y no tenían un tejido empresarial sólido, por lo que se apostó por incorporar valores, dar herramientas para escalar, estimular la creatividad y sus capacidades, así como fomentar la creación de redes». De forma complementaria, dentro del ecosistema de innovación social de MARES, se tejieron alianzas con las finanzas solidarias a través de la cooperativa de servicios financieros Coop 57 y el banco cooperativo Fiare Banca Etica. Esto permitió 
la concesión de microcréditos a iniciativas que en general tienen dificultad de acceso al crédito, como las microempresas vinculadas con la inserción social y laboral de personas, la agroecología, la eficiencia energética, la educación y la cultura, y el comercio justo, entre otras.

c) Clústeres sectoriales: espacios para experimentar, innovar y aprender

Un aspecto sobresaliente del proyecto es el aprovechamiento de infraestructuras públicas rehabilitadas y diseñadas como contenedores para albergar recursos y servicios sinérgicos para el impulso a la creatividad y la innovación social en diferentes sectores económicos. De este modo, MAR de Alimentación puso en marcha la primera cocina incubadora pública de la capital bajo el nombre Gastrolab Villaverde. Esta cocina industrial, con 150 metros cuadrados, daba apoyo a la consolidación y el desarrollo de iniciativas económicas del sector de la alimentación, en el que se acompañaron 20 proyectos desde su apertura a mediados de 2019. Desde el propio Gastrolab, se apostó por incrementar el valor añadido de los productos mediante la significación del atributo local, de la agricultura ecológica, de la transformación artesanal y del comercio justo. Lo más novedoso de este espacio fue poner a disposición de los usuarios la posibilidad de desarrollar tres líneas de producción con registro sanitario para su comercialización, lo que permitía a los emprendedores y las empresas incubadas vender sus productos con todas las garantías higiénico-sanitarias. De acuerdo con el director de la iniciativa, «esto no solo facilitó que nuevos emprendedores sin capacidad de invertir en una cocina con registro sanitario propio pudieran testear su oferta gastronómica antes de lanzarla al mercado», además, «el desarrollo de un espacio de referencia para un sector productivo concreto resultó fundamental para reforzar la proximidad geográfica y organizativa entre actores, lo que facilitó procesos que ayudaron a consolidar las iniciativas incubadas, incrementó la regularidad de los intercambios y favoreció la construcción de relaciones de confianza». En suma, se confirma la importancia de la proximidad para la generación de redes de cooperación entre empresas y para el establecimiento de vínculos comunitarios, condiciones necesarias para promover la innovación (Méndez, 2016), así como para la construcción de un sistema cooperativo adecuado en términos de desarrollo local (Perard, 2015).

\subsection{Caracteristicas y actividades de los usuarios del MAR de Alimentación}

La encuesta realizada permite contrastar los perfiles y las motivaciones de los usuarios del MAR de Alimentación, así como el uso que han hecho del espacio de Villaverde. Desde el punto de vista del perfil sociodemográfico, se confirma la relativa juventud de los usuarios, y destaca el peso de las mujeres, de los usuarios con estudios superiores y de los residentes en la ciudad de Madrid (tabla 1). Además, resulta claro el predominio de emprendedores que desarrollan un proyecto propio o que en el momento de contestar a la encuesta estaban en proceso de desarrollarlo (como estudiantes o desempleados). Cabe 
La innovación social como herramienta para hacer frente a los retos urbanos:

Tabla 1. Perfil sociodemográfico de los usuarios entrevistados

\begin{tabular}{|c|c|c|}
\hline \multicolumn{2}{|l|}{ Indicador } & Valor \\
\hline \multicolumn{2}{|l|}{ Edad media } & 42 años \\
\hline \multicolumn{2}{|l|}{ Porcentaje mujeres } & $59,5 \%$ \\
\hline \multicolumn{2}{|l|}{ Porcentaje nacidos en el extranjero } & $24,3 \%$ \\
\hline \multicolumn{2}{|l|}{ Porcentaje con estudios superiores } & $64,8 \%$ \\
\hline \multicolumn{2}{|l|}{ Residentes en la ciudad de Madrid } & $40,5 \%$ \\
\hline \multirow{4}{*}{$\begin{array}{l}\text { Situación laboral en el momento de } \\
\text { contactar con MARES-Alimentación }\end{array}$} & Desempleado o estudiando & $18,9 \%$ \\
\hline & Mismo sector por cuenta propia & $48,6 \%$ \\
\hline & Mismo sector por cuenta ajena & $5,4 \%$ \\
\hline & Distinto sector & $16,2 \%$ \\
\hline
\end{tabular}

Fuente: elaboración propia.

Tabla 2. Principales retos de los proyectos incubados

\begin{tabular}{lc}
\hline Retos & Menciones por parte de todos los usuarios \\
\hline Consumo saludable & 16 \\
Empleo de calidad & 6 \\
Soberanía alimentaria & 5 \\
Desarrollo sostenible & 4 \\
Mejorar la calidad de vida de la población & 3 \\
Reconectar vínculos campo-ciudad & 3 \\
Lucha contra el cambio climático & 2 \\
\hline
\end{tabular}

Fuente: elaboración propia.

relacionar este grupo con una motivación en principio «utilitarista» del espacio y un interés por los equipamientos y servicios ofrecidos. Esto no es incompatible con una motivación "militante». Así, más del 85\% de los usuarios dicen estar familiarizados con los paradigmas de la ESS, con la seguridad alimentaria o con ambos. Esto está en consonancia con la orientación de los proyectos incubados hacia determinados retos urbanos, identificados en común por los usuarios (tabla 2).

Para ahondar en la cuestión, en la tabla 3 se analizan las motivaciones a la hora de haberse acercado a MARES-Alimentación, según el grado de conocimiento de los mencionados paradigmas. Con carácter general, la principal motivación corresponde a la búsqueda de asesoramiento empresarial, seguida de la formación y el deseo de usar los recursos e infraestructuras disponibles o el desarrollo de innovaciones empresariales. La persecución de mayor impacto social o ambiental del proyecto o la ampliación de redes quedarían más rezagados. En este sentido, no existen diferencias significativas por grupos ${ }^{2}$, salvo en el caso precisamente de la ampliación de las redes, donde surge una respuesta

2. El limitado tamaño de la muestra disponible, especialmente en el caso de los usuarios que declaran no tener conocimiento de los paradigmas de referencia, no permite establecer diferencias estadísticamente significativas en los análisis exploratorios realizados. 
Tabla 3. Principales motivaciones para acercarse a MARES-Alimentación

\begin{tabular}{|c|c|c|c|c|}
\hline & \multirow[b]{2}{*}{$\begin{array}{l}\text { Valoración media por } \\
\text { parte de los usuarios } \\
\text { (escala: } 1 \text { mín.- } 5 \text { máx.) }\end{array}$} & \multicolumn{3}{|c|}{$\begin{array}{c}\text { Valoración media según grado de conocimiento } \\
\text { de los paradigmas }\end{array}$} \\
\hline & & $\begin{array}{c}\text { Ninguno } \\
\text { (usuarios: 5) }\end{array}$ & $\begin{array}{l}\text { co. social solidaria } \\
\text { o seguridad } \\
\text { alimentaria } \\
\text { (usuarios: } 14 \text { ) }\end{array}$ & $\begin{array}{c}\text { Ambos } \\
\text { (usuarios: 18) }\end{array}$ \\
\hline Asesoramiento empresarial & 4,3 & 5,0 & 4,1 & 4,2 \\
\hline Uso de recursos e infraestructuras & 3,8 & 4,0 & 3,3 & 3,2 \\
\hline Innovación empresarial del proyecto & 3,5 & 4,3 & 3,1 & 4,2 \\
\hline Ampliación o contacto con redes territoriales & 3,0 & 3,0 & 2,6 & 4,1 \\
\hline Formación y ampliar conocimiento & 3,9 & 3,7 & 3,8 & 4,4 \\
\hline Mejora impacto social y ambiental del proyecto & 3,3 & 4,0 & 2,4 & 4,0 \\
\hline
\end{tabular}

Fuente: elaboración propia.

ciertamente baja de los usuarios que sí conocen la ESS, quizás porque precisamente ya participan en ellas. En todo caso, la máxima diferencia entre la valoración de los usuarios con mayor conocimiento de los paradigmas y aquellos que dicen desconocerlos por completo es en cuanto a la ampliación de las redes.

Con relación a la cuestión de las redes, cabe preguntarse por el papel de la proximidad entre actores y la escala espacial en los procesos de innovación social. De los 37 usuarios entrevistados, un 64\% tiene estudios superiores, entre los que destaca el grupo de aquellos con estudios empresariales, por delante de ingenieros agrónomos, forestales o de caminos, así como de graduados en biología y ciencias ambientales. Cabe interpretar que cada uno de estos subgrupos presentaría mayor proximidad cognitiva. Por otro lado, un 54\% de los usuarios ya trabajaban en el sector de la alimentación, mayoritariamente por cuenta propia, lo que cabe vincular con una forma de proximidad relacional, al compartir a priori estos actores un espacio de relaciones similar.

La tabla 4 busca comparar estas formas de proximidad previa de cada usuario con el resto, con alguna variable de resultado que permita evidenciar su influencia sobre la colaboración e innovación. Sin perder de vista que muchas de las iniciativas estaban en sus primeros estadios de desarrollo, así como el retraso en la puesta en marcha de la infraestructura clave para el testeo de productos, un buen indicador de resultado puede ser la creación o ampliación de las redes entre iniciativas cohabitando en Villaverde. Los resultados apuntan a que efectivamente aquellos usuarios que presentan mayor proximidad cognitiva o relacional habrían visto ampliadas sus redes de colaboración en mayor medida que el resto. En concreto, de los 20 actores que al acercarse al MAR de Alimentación ya tenían un proyecto en marcha dentro del sector, el 55\% logró ampliar sus redes de colaboración. De los restantes 17 actores que desarrollaron un proyecto dentro del sector utilizando los recursos de MARES, más del 70\% ampliaron también sus redes. Esto apunta a esa proximidad construida derivada de la participación en las redes impulsadas desde esta infraestructura urbana. 
Tabla 4. Formas de proximidad entre usuarios y redes de conocimiento

\begin{tabular}{llcc}
\hline & & \multicolumn{2}{c}{$\begin{array}{c}\text { Usuarios que han ampliado } \\
\text { sus redes de conocimiento }\end{array}$} \\
\cline { 3 - 4 } & & Recuento & $\%$ \\
\hline Proximidad cognitiva por formación universitaria & Biología, ciencias ambientales & 3 & 100,0 \\
& Estudios de empresa, marketing & 5 & 100,0 \\
& Ingeniero montes, forestales, caminos & 2 & 40,0 \\
& Psicología, educación, trabajo social & 1 & 25,0 \\
& Otras titulaciones & 5 & 71,4 \\
& Sin estudios superiores & 7 & 53,8 \\
Proximidad relacional por sector de la iniciativa & Sector alimentación & 11 & 55,0 \\
& Otros sectores & 12 & 70,6 \\
\hline
\end{tabular}

Fuente: elaboración propia.

La consulta a los usuarios aporta igualmente evidencias sobre cómo la concentración de recursos y servicios en el espacio de MARES- Villaverde ha reforzado su consideración como polo de innovación de referencia dentro del ecosistema regional. Como evidencia inicial, cabe señalar que si bien un $43 \%$ de los encuestados son residentes en la ciudad de Madrid, otro 31\% vive en la región e incluso un 8,6\% lo hace en otra provincia. Estos usuarios acudirían al espacio para participar sobre todo en la cocina incubadora Gastrolab Villaverde, lo que pone de manifiesto la importancia estratégica de este equipamiento. Otras iniciativas principales destacadas serían las cooperativas de comerciantes y logística. Un porcentaje notable menciona la participación en otras iniciativas, como eventos, talleres o cursos (tabla 5).

Desde el punto de vista de los servicios, destaca el interés por los servicios de asesoramiento empresarial y de formación, que ciertamente pueden ser más

Tabla 5. Participación en iniciativas de MARES

\begin{tabular}{|c|c|c|c|c|}
\hline & \multirow[b]{2}{*}{$\begin{array}{l}\text { Menciones por parte } \\
\text { de los usuarios }\end{array}$} & \multicolumn{3}{|c|}{$\begin{array}{l}\text { Menciones según grado de conocimiento } \\
\text { de los paradigmas }\end{array}$} \\
\hline & & $\begin{array}{l}\text { Ninguno } \\
\text { (usuarios: } 5 \text { ) }\end{array}$ & $\begin{array}{l}\text { Eco. social } \\
\text { solidaria } 0 \\
\text { seguridad } \\
\text { alimentaria } \\
\text { (usuarios: 14) }\end{array}$ & $\begin{array}{c}\text { Ambos } \\
\text { (usuarios: 18) }\end{array}$ \\
\hline (Iniciativa principal) & Recuento & $\%$ & $\%$ & $\%$ \\
\hline Gastrolab Villaverde registro sanitario & 15 & 60,0 & 35,7 & 38,9 \\
\hline Cooperativa logística & 4 & 20,0 & 7,1 & 11,1 \\
\hline Cooperativa de comerciantes & 9 & 0 & 21,4 & 33,3 \\
\hline Supermercados cooperativos & 2 & 0 & 7,1 & 5,6 \\
\hline Huertos urbanos y comunitarios & - & - & - & - \\
\hline Otros & 7 & 20,0 & 28,6 & 11,1 \\
\hline
\end{tabular}

Fuente: elaboración propia. 


\begin{tabular}{lcccc}
\hline & & \multicolumn{3}{c}{$\begin{array}{c}\text { Menciones según grado de conocimiento } \\
\text { de los paradigmas }\end{array}$} \\
\cline { 3 - 5 } & & \multicolumn{3}{c}{$\begin{array}{c}\text { Eco. social } \\
\text { solidaria 0 } \\
\text { seguridad }\end{array}$} \\
\cline { 3 - 5 } & $\begin{array}{c}\text { Menciones por parte } \\
\text { de los usuarios }\end{array}$ & $\begin{array}{c}\text { Ninguno } \\
\text { (usuarios: 5) }\end{array}$ & $\begin{array}{c}\text { alimentaria } \\
\text { (usuarios: 14) }\end{array}$ & $\begin{array}{c}\text { Ambos } \\
\text { (usuarios: 18) }\end{array}$ \\
\hline Comunidades de aprendizaje & Recuento & $\%$ & $\%$ & $\%$ \\
Asesoramiento empresarial & 10 & 15,4 & 12,9 & 8,7 \\
Actividades formativas & 34 & 38,5 & 38,7 & 37,0 \\
Gastrolab (cocina) & 29 & 23,1 & 32,3 & 34,8 \\
\hline Fuente & 17 & 23,1 & 16,1 & 19,6 \\
\hline
\end{tabular}

Fuente: elaboración propia.

habituales en otros centros de innovación y emprendimiento más convencionales de la ciudad. En todo caso, el uso de esos servicios singulares en el contexto regional e incluso nacional, como son el Gastrolab o las CAP, es mencionado en más de la mitad de las respuestas (tabla 6). Con todo, se advierte una participación más activa en las distintas iniciativas y servicios por parte de aquellos usuarios con conocimiento de los paradigmas de referencia. Los que tendrían un interés más utilitarista participan también en ellas, pero en términos relativos su presencia se refuerza precisamente en actividades orientadas al asesoramiento empresarial, la formación o la innovación de producto en la cocina.

Como evidencia adicional, cabe señalar que, cuando observamos el ámbito geográfico del proyecto incubado o desarrollado desde MARES-Villaverde, advertimos que en un $40 \%$ de los casos el ámbito espacial de referencia de la iniciativa es la ciudad de Madrid, junto con otro $17 \%$ que, además de ese ámbito, tendría la región de Madrid. Los proyectos que se dirigen exclusivamente a la región de Madrid suponen otro 40\%, mientras que otro 3\% apuntaría a un ámbito fuera de la región.

\section{Conclusiones}

Desde el punto de vista del diseño del proyecto, el ecosistema de innovación social creado para el impulso y la generación de actividad económica en Madrid y su localización en una infraestructura concreta representa un punto de inflexión respecto a las políticas públicas urbanas de creación de empleo, con la introducción de factores de sostenibilidad y participación ciudadana. Las claves más innovadoras encontradas en el marco de esta investigación permiten contrastar las hipótesis planteadas.

En primer lugar, se confirma que el enfoque clúster promovió redes formales e informales de carácter horizontal entre diversos agentes en la escala local-regional en torno a los principios y valores de la ESS (administraciones 
públicas, emprendedores sociales, empresas, entidades de financiación ética y solidaria, investigadores, redes de ESS, tejido asociativo y la ciudadanía en general). La investigación ha confirmado que el enfoque sectorial aprovecha y potencia la proximidad entre actores, lo que da como resultado dinámicas de colaboración que van más allá de los procesos de innovación en productos y procesos dentro del sector, para orientarse a la transformación del modelo dominante y sentar con ello las bases de un desarrollo territorial más sostenible en la ciudad de Madrid. En el caso del MAR-Alimentación esto se refleja en la (co)existencia de una aspiración más «utilitarista» en el uso de los servicios y recursos, frente a otra más «militante», que tiene un protagonismo fundamental en la construcción de esas redes.

En este sentido, esta experiencia cumple con la perspectiva transformadora de la innovación social, abordada en el estado de la cuestión, al poner el énfasis en las acciones colectivas y donde la economía se rearticula a partir de la dimensión social y del arraigo con el territorio. Además, el conjunto de herramientas desarrolladoras de capacidades de innovación social formuladas en dicho ecosistema permitieron incidir en el impulso del conocimiento colectivo - tanto experto como práctico-, la incubación de nuevos proyectos con un marcado acento en la sostenibilidad y la articulación entre diferentes agentes de un sector productivo concreto. Todo ello representa una respuesta activa ante los desafíos producidos por una economía globalizada (precariedad, desigualdad, deslocalización, etcétera).

Finalmente, la concentración de recursos y servicios en los clústeres sectoriales favoreció que se convirtieran en espacios de interacción y de referencia a escala regional, para impulsar proyectos cooperativos destacados, estimular la creatividad, abrir nuevos debates en la agenda política y atraer a diversos actores, con intereses convergentes, de otros territorios fuera de la ciudad de Madrid. En concreto, el edificio de Villaverde adquiere una relevancia crítica en el caso de estudio analizado.

Asimismo, los resultados de esta investigación permiten reflexionar sobre las principales debilidades y aciertos detectados, de interés para otras iniciativas innovadoras de esta naturaleza. En primer lugar, gracias a la red de contactos y la experiencia de las entidades de ESS que coordinaban MARES, se ofreció un asesoramiento personalizado y un acompañamiento a la carta. Como resultado, se avanzó en mejorar los procesos de innovación empresarial y productiva. También las diferentes herramientas orientadas a la formación-acción, como las CAP, que favorecieron la implicación de otros agentes diferentes a los emprendedores sociales, contribuyeron a enriquecer el propio ecosistema de innovación social. El enfoque sectorial (alimentación, movilidad, reciclaje, energía y economía de los cuidados) se configuró como una apuesta estratégica para transformar el metabolismo urbano de Madrid, lo que sirvió para atraer a personas que aspiraban a construir un modelo de ciudad más sostenible y cohesionado social y territorialmente.

Con relación a las debilidades, cabe comenzar apuntando a la compleja relación con las instituciones públicas, con la existencia visible de dos «almas» 
desde el origen mismo del proyecto (ayuntamiento vs. redes de ESS). Además, el proyecto se vio afectado por las disputas en la política municipal, y por ello MARES se asoció a un proyecto ideologizado. Esto contribuyó a crear incertidumbre entre los responsables del proyecto y usuarios ante un posible cambio político, cuestión que dificultaba el diseño de estrategias a largo plazo. De hecho, la continuidad de MARES terminó en las elecciones municipales de 2019. Esto supuso no solo una pérdida de recursos humanos, conocimiento y costes económicos muy importantes, sino también la ruptura de una dinámica creciente de fortalecimiento de la ESS en las políticas públicas de Madrid. En definitiva, se truncó el proceso de adaptación y difusión de las soluciones de innovación social que se experimentaron de forma piloto en MARES.

A ello contribuyó el poco tiempo transcurrido entre la rehabilitación de los espacios públicos en desuso (requisito obligatorio de la convocatoria Urban Innovation Actions) y su puesta en marcha, lo que limitó los resultados en materia de creación de empleo y pudo además provocar cierta degradación de los ecosistemas sectoriales creados, de las conexiones y sinergias entre las nuevas empresas, los emprendedores y los diversos beneficiaros. Junto con ello, otra debilidad fue la apuesta del ayuntamiento por localizar los clústeres en barrios vulnerables con el objeto de reducir los desequilibrios territoriales en ellos. Esta apuesta parecía responder más a objetivos políticos que a una lógica de clúster tal y como se configuró el proyecto. En este sentido, la investigación en MARES-Alimentación ha confirmado que muchos proyectos tenían un ámbito de intervención diferente al distrito, sin olvidar además los problemas derivados de la localización en Villaverde, señalados por algunos usuarios.

Pese a estas cuestiones, el proyecto MARES en su conjunto ha contribuido a que Madrid disponga de unas novedosas infraestructuras públicas adecuadas para el fortalecimiento del tejido económico sectorial y para crear una nueva cultura empresarial. Ahora bien, la iniciativa corre el riesgo de que, si no se aplican en su gestión los valores y principios de una economía social y solidaria, participativa y sostenible, pierda todo su potencial para transformar el modelo económico en la ciudad de Madrid.

\section{Referencias bibliográficas}

Amin, Ash y Roberts, Joanne (2008). «Knowing in action: Beyond communities of practice». Research Policy, 37 (2), 353-369. <https://doi.org/10.1016/j.respol.2007.11.003>

AyunTAMIENTO De MADRID (2016). Estrategia de economía social y solidaria de la ciudad de Madrid 2017-2025. Madrid: Ayuntamiento de Madrid.

Balland, Pierre Alexandre; Boschma, Ron y Frenken, Koen (2015). «Proximity and Innovation: From Statics to Dynamics». Regional Studies, 49 (6), 907-920. $<$ https://doi.org/10.1080/00343404.2014.883598>

Bathelt, Harald; Malmberg, Anders y Maskell, Peter (2004). "Clusters and knowledge: Local buzz, global pipelines and the process of knowledge creation». Progress in Human Geography, 28 (1), 31-56. <https://doi.org/10.1191/0309132504ph469oa> 
Blanco, Ismael; Cruz Gallach, Helena; Martínez Moreno, Rubén y Parés, Marc (2016). «El papel de la innovación social frente a la crisis». Ciudady Territorio. Estudios Territoriales, 188, 249-260.

Boschma, Ron A. (2005). «Proximity and innovation: A critical assessment». Regional Studies, 39 (1), 61-74. <https://doi.org/10.1080/0034340052000320887>

Comité de las Regiones, UE (2002). «Dictamen. Asociación entre las autoridades locales y regionales y las organizaciones socioeconómicas: contribución al empleo, al desarrollo local y a la cohesión social». Diario Oficial de las Comunidades Europeas, 2002/C 192/13.

Conill, Joana; Cárdenas, Amalia; Castells, Manuel; Servon, Lisa y Hlebik, Svetlana (2012). Otra vida es posible. Prácticas económicas alternativas durante la crisis. Barcelona: UOC.

Espinosa, Ana (2019). «El papel de los grupos de consumo agroecológico en la construcción de un sistema de distribución y un orden alimentario alternativos». En: SÁnChez Hernández, J. L. (ed.). Espacios y prácticas económicas alternativas en las ciudades españolas. Navarra: Thomson Reuters Aranzadi, 65-86.

Fløysand, Arnt y Jakobsen, Stig-Erik (2011). "The complexity of innovation: A relational turn». Progress in Human Geography, 35 (3), 328-344. <https://doi.org/10.1177/0309132510376257>

Fuente, Rosa de la (2019). Alianzas locales para los retos globales. Colección de Cuadernos para la Internacionalización de las Ciudades. CDMX - UCCI - Madrid: Universidad Complutense de Madrid. Recuperado de <https://www.acimedellin. org/wp-content/uploads/2019/10/cuaderno-allas-09.pdf>.

Fuller, Duncan y Jonas, Andrew (2003). «Alternative Financial Spaces». En: LeYsHON, Andrew et al. (eds.). Alternative Economic Spaces. Londres: SAGE, 55-73.

Gibson-Graham, J. Katherine (2008). «Diverse economies: Performative practices for "other worlds"”. Progress in Human Geography, 32 (5), 613-632. <https://doi.org/10.1177/0309132508090821>

González-Romero (2020). «La innovación social como estrategia de desarrollo. Políticas urbanas y acción colectiva». TEUKEN BIDIKAY. Revista Iberoamericana de Investigación en Organizaciones, Ambiente y Sociedad, 11 (16), 29-54.

Guridi, Luis y Pérez De Mendiguren, Juan Carlos (2014). La dimensión económica del desarrollo humano local: la economía social y solidaria. Universidad del País Vasco-HEGOA. Recuperado de <https://publicaciones.hegoa.ehu.eus/uploads/ pdfs/265/Economia_social_y_solidaria_DHL.pdf?1488539833>.

KLEIN, Juan-Luis (2019). «Innovación social y análisis territorial. Los nuevos retos». En: Michelini, Juan José (ed.). La metrópolis creativa. Innovaciones sociales en América Latina y el sur de Europa. Madrid: Catarata, 84-102.

Klein, Juan-Luis; Fontan, Jean-Marc; Harrisson, Denis y Lévesque, Benoît (2014). «L'innovation sociale au Québec: un système d'innovation fondé sur la concertation». En: Klein, Juan-Luis; Laville, Jean Louis y Moulaert, Frank (eds.). L'innovation sociale. Toulouse: ÉRÈS, 193-246.

Laville, Jean-Louis (2009). "La economía solidaria en perspectiva». En: Laville, Jean-Louis y Garcia Jané, Jordi (eds.). Crisis capitalista y economía solidaria. Una economía que emerge como alternativa real. Barcelona: Icaria Editorial, 63-128.

LÉVESQUE, Benoît (2011). «Innovations sociales et pouvoirs publics: vers un système québécois d'innovation dédié à l'économie sociale et solidaire. Quelques éléments de problématique». CRISES, Collection Études Théoriques, 9-25. 
Leyshon, Andrew; Lee, Roger y Williams, Colin C. (2003). Alternative Economic Spaces. Londres: SAGE Publications Ltd. <https://doi.org/10.4135/9781446220825>

Llobera, Franco (2013). «Transiciones ecológicas y desarrollo local». Retos y Futuro del Desarrollo Económico Local. Red de Entidades para el Desarrollo Local (REDEL), 171-183.

Maillat, Denis (1992). "La relation des entreprises innovatrices avec leur milieu». En: Maillat, Denis y Perrin, Jean-Claude (eds.). Entreprises innovatrices et développement territorial. Neuchâtel: EDES, 3-22.

MARES MADRID (2019). Memoria final proyecto MARES Madrid 2016-2019. Madrid: MARES Madrid.

Martínez Moreno, Rubén (2011). Políticas públicas e innovación social. Marcos conceptuales y efectos en la formulación de las politicas. Documento de trabajo. UAB.

Mason, Paul (2015). Postcapitalism. A guide to our future. Londres: Penguin Books.

Méndez, Ricardo (2016). «Renovar economías urbanas en crisis: un debate actual sobre la innovación». Desenvolvimento Regional em Debate, 6 (3), 4-31.

- (2018). «Redes económicas alternativas. ¿Para una sociedad postcapitalista? Algunas experiencias en Madrid». XV Coloquio Internacional de Geocritica. Las ciencias sociales y la edificación de una sociedad post-capitalista. GEO crítica y Universitat de Barcelona.

MÉndez, Ricardo y SánChez-Moral, Simón (2011). «Spanish cities in the knowledge economy: theoretical debates and empirical evidences». European Urban and Regional Studies, 18 (2), 136-155. <https://doi.org/10.1177/0969776410381039>

MéndeZ, Ricardo y Monteserín, Obdulia (2017). «Redes alimentarias alternativas en grandes ciudades: los mercados de productores agrarios en Madrid». Cuadernos Geográficos, 56 (1), 193-216.

Méndez Gutiérrez del Valle, Ricardo; Sánchez Moral, Simón; Abad AraGón, Luis y García Balestena, Ignacio (2009). "Sistema urbano y sociedad del conocimiento: hacia una tipología de las ciudades españolas». Investigaciones Regionales, 16, 117-142.

Michelini, Juan José (2019). «Innovaciones sociales en Europa y América Latina: un estado de la cuestión». En: Michelini, Juan José (ed.). La metrópolis creativa. Innovaciones sociales en América Latina y el sur de Europa. Madrid: Catarata, 27-83.

Moulaert, Frank y Nussbaumer, Jacques (2005). «Defining the social economy and its governance at the neighbourhood level: A methodological reflection». Urban Studies, 42 (11), 2.071-2.088. <https://doi.org/10.1080/420980500279752>

Moulaert, Frank y Sekia, Farid (2003). «Territorial innovation models: A critical survey». Regional Studies, 37 (3), 289-302. <https://doi.org/10.1080/0034340032000065442>

Moulaert, Frank; Martinelli, Flavia; González, Sara y Swyngedouw, Erik (2007). «Introduction: Social innovation and governance in European cities: Urban development between path dependency and radical innovation». European Urban and Regional Studies, 14 (3), 195-209. <https://doi.org/10.1177/0969776407077737>

Navarro, Clemente; Parés, Marc; Martí-Costa, Marc; Díaz, Fernando; WalliSER, Andrés; Rodríguez, María Jesús y VÁzquez, Octavio (2019). Innovación en politicas urbanas. Perspectivas, metodologías y casos. Barcelona: Icaria Editorial. 
NeL.LO, Oriol (2015). La ciudad en movimiento: crisis social y respuesta ciudadana. Madrid: Díaz \& Pons Editores.

Perard, Pierre (2015). «Les clusters d'innovation sociale. Analyse et bonnes pratiques européennes». Pour la Solidarité. Notes d'Analyse, 19.

Pérez de Mendiguren, Juan Carlos; Etxezarreta Etxarri, Enekoitz y Guridi AldaNONDO, Luis (2009). «Economía Social, Empresa Social y Economía Solidaria: diferentes conceptos para un mismo debate». Papeles de Economía Solidaria, 1, 13-18.

Polanyi, Karl (1944). La gran transformación. Los orígenes politicos y económicos de nuestro tiempo. México: Fondo de Cultura Económica.

Salom, Julia; Pitarch, María Dolores y Sales, Ana (2017). «Innovación social: estrategias urbanas en un contexto de cambio. El caso de la ciudad de Valencia». CIRIEC-España Revista de Economía Publica, Social y Cooperativa, 91, 31-58. <https://doi.org/10.7203/CIRIEC-E.91.10451>

Salom, Julia; Pitarch, María Dolores; Sales, Ana y Cornadó, Marc (2019). «Prácticas económicas alternativas e innovación social: el caso de la ciudad de Valencia». En: Sánchez Hernández, J. L. (ed.). Espacios y prácticas económicas alternativas en las ciudades españolas. Navarra: Thomson Reuters Aranzadi, 165-184.

SÁnCHez Hernández, José Luis (coord.) (2019). Espacios y prácticas económicas alternativas en las ciudades españolas. Navarra: Thomson Reuters Aranzadi.

Subirats, Joan y García Bernardos, Ángela (eds.) (2015). Innovación social y políticas urbanas en España. Experiencias significativas en las grandes ciudades. Icaria Política, 2. Barcelona: Icaria Editorial.

Suriñach, Ruben (2017). Economies transformadores de Barcelona. Barcelona: Montaber-Ajuntament de Barcelona.

Vázquez Barquero, Antonio (1999). Desarrollo, redes e innovación. Madrid: Pirámide.

Walliser, Andreas y Fuente, Rosa de la (2018). «Los nuevos activismos urbanos y la institucionalidad del cambio en Madrid». En: Baron-Yelles, Nacima y Romero, Joan (eds. lits.). Cultura territorial e innovación social. ¿Hacia un nuevo modelo metropolitano en Europa del Sur? Valencia. Universidad de Valencia, 95-114.

Wenger, Etienne (2001). «Una teoría del aprendizaje. Comunidades de práctica: aprendizaje, significado e identidad». Barcelona: Paidós. Recuperado de <http://cmap.javeriana.edu.co/servlet/SBReadResourceServlet? rid=1JP2KX093$1 \mathrm{GX} 1 \mathrm{ZY0} 0-28 \mathrm{~S}>$.

Yacamán, Carolina; Ruiz Salgado, Antonio y Navarro, Alberto (2018). Entidades sin ánimo de lucro y la economía social y solidaria. Formas de trabajo para la conservación de la naturaleza y la gestión de los recursos naturales. Madrid: Asociación de Fundaciones para la Conservación de la Naturaleza y Fundación Biodiversidad Ministerio de Agricultura y Pesca, Alimentación y Medio Ambiente.

Yacamán, Carolina; Mata, Rafael; Matarán, Alberto; López, José María y FuentesGuerra, Rafael (2019). "The Potential Role of Short Food Supply Chains in Strengthening Periurban Agriculture in Spain: The Cases of Madrid and Barcelona». Sustainability, 11 (7), 1-19. <https://doi.org/10.3390/su11072080>

Yacamán, Carolina; Mata, Rafael y Matarán, Alberto (2020). «Los sistemas territoriales de innovación social para mejorar la viabilidad de la agricultura periurbana. El caso de estudio de la región urbana de Madrid». En: Farinos, Joaquín (coord.) et al. (eds.). Desafios y oportunidades de un mundo en transición. Una interpretación desde la geografía. Valencia: Publicacions de la Universitat de València, 317-330. 
